\title{
Cuidado em saúde à pessoa vivendo com HIV/AIDS: representações sociais de enfermeiros e médicos
}

\author{
Health care for people living with HIV/AIDS: social representations among nurses \\ and doctors
}

\section{Cuidados de salud a la persona portadora de VIH / SIDA: representaciones sociales de enfermeros y médicos}

\author{
Virginia Paiva Figueiredo Nogueira'; Antonio Marcos Tosoli Gomes ${ }^{I I}$; \\ Yndira Yta Machado ${ }^{\text {III; }}$ Denize Cristina de Oliveira ${ }^{\text {IV }}$
}

\begin{abstract}
RESUMO: Este estudo objetivou identificar as representações sociais de enfermeiros e médicos acerca do cuidado em saúde à pessoa vivendo com o Vírus da Imunodeficiência Humana (HIV) e a Síndrome da Imunodeficiência Adquirida (AIDS). Trata-se de um estudo descritivo com abordagem quantiqualitativa, orientado pela Teoria das Representações Sociais em sua abordagem estrutural. Foram cenários deste estudo, 18 unidades de saúde no município do Rio de Janeiro, especializadas na assistência em HIV/AIDS, entre 2011 e 2012. Participaram 54 médicos e 27 enfermeiros que responderam ao questionário de evocações, analisados posteriormente, com o auxílio do software EVOC. Como resultado, destaca-se o núcleo central das representações desses profissionais, formado por elementos positivos, como acolhimento, informação, adesão-tratamento e futuro, mas foi observado que médicos e enfermeiros mostram diferenças na representação do objeto estudado. Conclui-se que os profissionais revelaram uma representação positiva do objeto estudado, significando-o com base no saber científico e nas recomendações do Ministério da Saúde. Palavras-Chave: Representações sociais; cuidado em saúde; HIV; AIDS.
\end{abstract}

ABSTRACT: This study aimed to identify nurses' and doctors' social representations of health care for people living with Human Immunodeficiency Virus and Acquired Immunodeficiency Syndrome (HIV/AIDS). This quantitative and qualitative descriptive study was guided by the structural approach to social representations theory. Eighteen health institutions in Rio de Janeiro, specializing in care for people with HIV/AIDS, were the scenarios for this study in 2011 and 2012. The participants were 54 doctors and 27 nurses, who answered the evocation questionnaire, which was subsequently analyzed using EVOC software. Salient results include these professionals' core representations, comprising positive elements, such as user embracement, information, treatment adherence and future. However, doctors and nurses were found to differ in their representations of the study object. It was concluded that professionals have a positive image of such care, signifying it on the basis of scientific knowledge and Ministry of Health recommendations. Keywords: Social representations; healthcare; HIV; AIDS.

RESUMEN: Este estudio ha objetivado identificar las representaciones sociales de enfermeros y médicos sobre el cuidado en salud a la persona portadora del Virus de la Inmunodeficiencia Humana $(\mathrm{VIH})$ y el Síndrome de Inmunodeficiencia Adquirida (SIDA). Se trata de un estudio descriptivo, de abordaje cuantitativo/cualitativo, orientado por la teoría de las representaciones sociales en su enfoque estructural. Han sido escenarios de este estudio: 18 centros de salud en Río de Janeiro, especializados en la asistencia del VIH/SIDA entre 2011 y 2012. Han participado 54 médicos y 27 enfermeros que contestaron el cuestionario de evocaciones, posteriormente analizado con la ayuda del software EVOC. Como resultado destaca el núcleo central de las representaciones de estos profesionales, compuesto de elementos positivos, como: acogida, información, adhesión/tratamiento y futuro, sin embargo se ha observado que los médicos y enfermeros presentan diferencias en la representación del objeto estudiado. Se concluye que los profesionales revelaron una representación positiva del objeto estudiado, significándolo teniendo como base el conocimiento científico y las recomendaciones del Ministerio de Salud.

Palabras Clave: Representaciones sociales; cuidado de salud; VIH; SIDA.

\section{INTRODUÇÃO}

Este estudo apresenta resultados da dissertação $\mathrm{O}$ cuidado em saúde à pessoa que vive com HIV/ AIDS: representações sociais de enfermeiros e médicos e integra o estudo multicêntrico As transformações do cuidado de saúde e enfermagem em tempos de AIDS: representações sociais e memórias de enfermeiros e profissionais de saúde no Brasil.

Na década de 1980, quando surgiu o primeiro caso de Síndrome da Imunodeficiência Adquirida (AIDS) nos Estados Unidos, cientistas, profissionais

\footnotetext{
IEnfermeira. Mestre em enfermagem pela Universidade do Estado do Rio de Janeiro. Rio de Janeiro, Brasil. E-mail: virginiafigueiredo@yahoo.com.br. IIEnfermeiro. Doutor em Enfermagem pela Universidade Federal do Rio de Janeiro. Professor Titular, Departamento de Enfermagem Médico-Cirúrgica e Coordenador do Programa de Pós-Graduação em Enfermagem da Universidade do Estado do Rio de Janeiro. Brasil. E-mail: mtosoli@gmail.com. IIIEnfermeira. Mestranda em enfermagem pela Universidade do Estado do Rio de Janeiro. Brasil. E-mail: yndiramachado@gmail.com.

IVEnfermeira. Doutora em Saúde Pública pela Universidade de São Paulo. Professora Titular, Departamento de Fundamentos de Enfermagem da Faculdade de Enfermagem da Universidade do Estado do Rio de Janeiro. Brasil. E-mail: dcouerj@gmail.com.
} 
da saúde e a população desconheciam tal doença e muito menos o seu agente etiológico, o que gerou pânico nas pessoas, inclusive nos profissionais que não sabiam como tratar os indivíduos que contraíam o Vírus da Imunodeficiência Humana (HIV), pelo medo da contaminação. Nesse período, as autoridades sanitárias supunham que as doenças infecciosas estavam controladas, em função das tecnologias e do saber médico da época, despertando comportamentos e respostas coletivos relacionados ao medo do contágio e de uma doença desconhecida. No Brasil, a epidemia evoluiu demonstrando contradições sociais, econômicas e culturais ${ }^{1}$.

A situação social e moral que surgiu com o advento da epidemia de AIDS, associada aos efeitos da doença, uma vez que seu desconhecimento médico era acompanhado do desconhecimento social, favoreceram o surgimento de representações que circularam por veículos de comunicação e por meio das conversas. Alguns qualificavam a AIDS como consequência da decadência moral ou castigo de Deus, o que a tornou um estigma social, provocando rejeição e gerando, nos excluídos, revolta ou submissão ${ }^{2}$.

O vírus e a síndrome, desconhecidos, constituíram os elementos essenciais para a formação de representações sociais, sendo transformados em algo conhecido, HIV/AIDS, sendo possível de serem pensados e passíveis de ação. Desde então, emergiram diversas representações da síndrome e das pessoas por ela acometidas. Estas foram modificadas, influenciadas pelas mudanças epidemiológicas, pela organização social e pelo desenvolvimento científico ${ }^{3}$.

Com o avanço das pesquisas, descobriu-se a causa da AIDS, desenvolveram-se testes para detecção do vírus, mecanismos de prevenção, autoproteção profissional e medicamentos antirretrovirais. Mas ressalta-se que os sentimentos dos pacientes durante a descoberta da sua soropositividade ao HIV são de dor e sofrimento, tornando bastante difícil o atendimento a este cliente, tanto para ele quanto para o profissional, já que este diagnóstico está interligado aos sentimentos de morte, perda, abandono, preconceito, rejeição pelas outras pessoas, rompimento das relações e medo do desconhecido ${ }^{4}$.

Em 1996, o Brasil promulgou a Lei $n^{\circ} 9.313$, de 13 de novembro de 1996, que dispõe sobre a distribuição gratuita de medicamentos para todas as pessoas acometidas pelo HIV/AIDS, mesmo contra as recomendações e as advertências do Banco Mundial ${ }^{5}$. As políticas públicas vigentes no país em relação à epidemia da AIDS têm como foco prestar assistência de qualidade às pessoas que convivem com o HIV/AIDS. Nesse sentido, foram ampliados os serviços assistenciais e credenciados hospitais para seu atendimento.

Ainda hoje, o contágio pelo HIV abarca, aproximadamente, 2,1 milhões de novas pessoas infectadas no mundo ${ }^{6}$. É importante destacar a importância da prevenção das doenças e a promoção da saúde que, com ações educativas, tende a gerar mais consciência acerca da responsabilidade pela saúde, em especial nos jovens que já nasceram em tempos de AIDS, com tratamento acessível e uma convivência possível com o HIV.

Há dificuldades que interferem no cuidado às pessoas que vivem com HIV, destacando-se os conflitos éticos e medo de contágio por parte dos profissionais ${ }^{7}$. Então, é importante adotar uma abordagem interdisciplinar entre os profissionais, para que, ao surgirem dificuldades em conduzir situações que julguem de difícil resolução para si, sintam-se à vontade para encaminhar o paciente a outro profissional, membro da equipe. Com isso, permite-se que o cliente fale sobre seus sentimentos, sobre suas dúvidas e obtenha orientações e informações sobre o assunto, pois é principalmente no aconselhamento que o profissional consegue desempenhar essas atividades e todas as oportunidades de esclarecimento de dúvidas à pessoa que vive com HIV/AIDS (PVHA) e a seus familiares, ações de fundamental importância ${ }^{4}$.

Dessa forma, o cuidado em saúde consiste numa relação estabelecida entre pessoas, tendo em vista o alcance da melhoria do estado de saúde e da qualidade de vida. Assim sendo, as práticas de cuidado realizadas pelos profissionais não podem se restringir apenas à técnica, mas devem se expressar por meio das atitudes e do relacionamento com o outro a quem o cuidado é oferecido ${ }^{8}$.

Acredita-se que a investigação das representações sociais do cuidado para os enfermeiros e médicos que atuam com esta clientela pode ajudar a compreender como o cuidado é visto e realizado, assim como pode auxiliar no entendimento sobre as relações entre os profissionais e PVHA.

Este estudo questiona quais são as representações sociais do cuidado em saúde para enfermeiros e médicos. Propõe-se como objeto de estudo conhecer como estes profissionais representam o cuidado em saúde à pessoa que vive com HIV/AIDS. Como objetivo, definiu-se identificar as representações sociais de médicos e enfermeiros acerca do cuidado em saúde a esse cliente.

\section{Referencial Teórico-Metodológico}

A Teoria das Representações Sociais (TRS), em sua abordagem estrutural, confere ênfase à estruturação dos conteúdos cognitivos das representações, mas se ocupa também do processo de sua transformação a partir das práticas sociais ${ }^{9}$. As representações sociais são uma forma de conhecimento elaborado e partilhado pela sociedade com uma visão prática para a construção de uma realidade comum a um conjunto social ${ }^{2}$.

A abordagem estrutural organiza a representação social em dois sistemas: o núcleo central, constituído por um ou alguns elementos cognitivos, ligados à memória coletiva e à história do grupo que formou a representação. Apresenta-se como consensual, coerente, estável, resistente à mudança e pouco sensível ao contexto social 
imediato, gerando o sentido básico da representação e organizando-a como um todo. O sistema periférico é constituído por todos os demais elementos da representação e caracteriza-se por integrar as experiências e as histórias individuais, suportar a heterogeneidade e as contradições do grupo. Além disso, é capaz de mudar mais facilmente em resposta às transformações do contexto social imediato e desempenha as funções de adaptação da representação à realidade concreta, de regulação flexível do seu conteúdo e de proteção do sistema central contra a possibilidade de mudanças frequentes ${ }^{10}$.

Este é um estudo descritivo com abordagem quantiqualitativa, orientado pela teoria das representações sociais em sua abordagem estrutural. O cenário de estudo foi composto por 18 unidades de saúde, nas quais são desenvolvidas atividades baseadas nas determinações da Coordenação Nacional Doenças Sexualmente Transmissíveis (DST), AIDS e Hepatites Virais do Ministério da Saúde, escolhidas de forma aleatória, de modo que abrangessem todas as áreas programáticas do município do Rio de Janeiro. Entre os serviços em que os participantes do estudo trabalhavam, ressaltamse: Centro de Testagem Anônima, Centro de Referência em HIV/AIDS, ambulatório especializado e serviços que foram agrupados como outros (ambulatório de imunologia, enfermaria de doenças infecto-parasitárias, hospital-dia, sala de procedimento da clínica médica e atendimento à tuberculose).

Os participantes foram 27 enfermeiros e 54 médicos, totalizando 81 profissionais que responderam ao questionário socioprofissional e às evocações, livres ao termo indutor Cuidar da pessoa com HIV/AIDS. $\mathrm{Na}$ coleta das evocações, foi solicitado que os profissionais falassem as cinco primeiras palavras que viessem a sua mente ao ouvirem o termo indutor.

Os dados do questionário socioprofissional foram organizados com o auxílio do software SPSS e analisados com o auxílio da estatística descritiva. Para a análise das evocações, foi utilizado o quadro de quatro casas do software EVOC 2005, buscando identificar a estruturação dos conteúdos representacionais.
A participação da pesquisa foi voluntária, a partir da aceitação dos profissionais diante do conhecimento dos objetivos da pesquisa. Os encontros foram realizados nos locais de trabalho e a coleta dos dados ocorreu no município do Rio de Janeiro, no período de agosto de 2011 a julho 2012. Foram respeitadas as normas e diretrizes de realização das pesquisas envolvendo seres humanos, segundo a Resolução no 196/96, vigente no período do planejamento do estudo. O projeto foi apresentado ao Comitê de Ética da Universidade do Estado do Rio de Janeiro, sendo aprovado com número de protocolo 048.3.2010; também foi utilizado um Termo de Consentimento Livre e Esclarecido, assinado pelos participantes do estudo.

\section{Resultados e Discussão}

Os participantes eram, em sua maioria, do sexo feminino (68\%), com idades que variavam entre as faixas etárias de até 35 anos (32,10\%) e de 46 a 55 anos $(34,57 \%)$, viviam com companheiro $(50,61 \%)$, professavam a religião católica $(49,38 \%)$, possuíam especialização $(62,96 \%)$, trabalhavam em ambulatório especializado em HIV/AIDS (67,96\%), referiram atualizar-se por meio de periódicos, livros, capacitações e congressos $(49,7 \%)$ e trabalhavam com DST/AIDS entre um a cinco anos $(30,8 \%)$.

Como resultado, foram obtidas 377 palavras evocadas, entre as quais 113 diferentes. Considerando o dicionário das palavras que foram evocadas pelos participantes, o software EVOC calcula a frequência simples de ocorrência de cada palavra evocada, média ponderada da ocorrência e ordens médias. A partir dessas informações, foi definido como ponto de corte a frequência mínima de 9 , tendo sido calculadas a frequência média - 12 - e a ordem média de evocação (OME) - 2,90 -, que corresponde à média de posição de cada palavra no corpus analisado. Tal critério foi utilizado conforme a Lei de Zpif de distribuição de palavras ${ }^{11}$.

Os resultados da análise de evocações ou prototípica do conjunto de profissionais do município estudado são apresentados na Figura 1.

\begin{tabular}{|c|c|c|c|c|c|c|}
\hline OME & $<2,9$ & & & $>=2,9$ & & \\
\hline $\begin{array}{l}\text { Freq } \\
>=12\end{array}$ & $\begin{array}{l}\text { Acolhimento } \\
\text { Informação } \\
\text { Adesão-tratamento } \\
\text { Futuro }\end{array}$ & $\begin{array}{c}\text { Freq. } \\
14 \\
14 \\
13 \\
12\end{array}$ & $\begin{array}{r}\text { OME } \\
2,28 \\
2,64 \\
2,69 \\
2,75\end{array}$ & Amor & $\begin{array}{l}\text { eq. } \\
18\end{array}$ & $\begin{array}{r}\text { OME } \\
3,5\end{array}$ \\
\hline $\begin{array}{l}\text { Freq } \\
<12\end{array}$ & $\begin{array}{l}\text { Cuidado } \\
\text { Atenção } \\
\text { Educação-saúde }\end{array}$ & $\begin{array}{c}\text { Freq } \\
11 \\
10 \\
10\end{array}$ & $\begin{array}{l}\text { OME } \\
2,36 \\
2,50 \\
2,80\end{array}$ & $\begin{array}{l}\text { Paciência } \\
\text { Tratamento } \\
\text { Aconselhamento } \\
\text { Tratamento- medicamentoso }\end{array}$ & $\begin{array}{c}\text { Freq. } \\
10 \\
9 \\
9 \\
9\end{array}$ & $\begin{array}{l}\text { OME } \\
3,00 \\
3,11 \\
3,22 \\
3,22 \\
\end{array}$ \\
\hline
\end{tabular}

FIGURA 1: Quadro de quatro casas referente às evocações de enfermeiros e médicos ao termo indutor cuidar da PVHA, Rio de Janeiro, 2014 ( $n=81$ sujeitos, Fmín = 9; Rang médio = 2,9; Fméd = 12). 
Os dados apresentados permitem apontar, como constituintes do possível núcleo central, as palavras acolhimento, informação, adesão-tratamento e futuro, que refletem a estruturação do processo assistencial no seio das determinações da Coordenação de DST, AIDS e Hepatites Virais, tratando-se de sua retradução na prática de cuidado. Destacam-se, ainda, questões ligadas a este núcleo: a representação social organiza-se ao redor do processo temporal de atendimento, que começa no acolhimento e visa ao aumento da sobrevida, especialmente na atualidade em que a AIDS existe como um quadro crônico, perpassando o repasse de informações, o processo de adesão e tratamento; e a representação mostra-se tensionada entre as recomendações do Ministério da Saúde, como já pontuado, e as características de cada profissional, como o zelo no acolhimento, a base científica e a eficácia frente ao processo saúde-doença (Figura 1).

No quadrante inferior esquerdo, correspondente à zona de contraste, foram identificados cuidado, atenção e educação-saúde, termos que reforçam o núcleo central e se apresentam como seu desdobramento, destacando o léxico cuidado, que se mostra como uma dimensão imagética da representação. No quadrante superior direito, primeira periferia, aparece o cognema amor, o qual indica a dimensão da afetividade e expressa atitude de cuidado e de preocupação pelo outro, características das profissões que atuam na área da saúde.

No quadrante inferior direito, segunda periferia, foram identificadas as palavras paciência, tratamento, aconselhamento e respeito, revelando a dimensão prática do cuidado, por meio da qual a PVHA poderá ter uma melhor condição de vida.

Identificaram-se elementos, como prováveis componentes do sistema central da representação, que estão relacionados às ações e recomendações oficiais do Ministério da Saúde e às características essenciais das profissões de medicina e enfermagem, todos eles positivos. Os resultados evidenciam também uma representação do cuidado relacionada à ética profissional, comprometimento com a vida e com a promoção da saúde. Ressalta-se, ainda, a importância de realizar estudos que reduzam a pressão normativa para uma melhor compreensão do próprio objeto.

Buscando-se um melhor entendimento sobre o grupo estudado, foi realizada a análise comparativa entre os profissionais que integraram o estudo, procedendo a uma análise EVOC, separadamente, das evocações livres de médicos e de enfermeiros. De acordo com a evocação dos médicos $(n=54)$, o software EVOC 2005 identificou um total de 246 palavras evocadas, das quais 92 eram diferentes. A frequência mínima foi de 6 , a frequência média de 9 e a OME, 2,80. Quanto à evocação dos enfermeiros $(n=27)$, o software identificou um total de 131 palavras, sendo que 63 eram diferentes. A frequência mínima foi de 4, a média, de 6 e a OME alcançou 2,90. A partir do quadro de quatro casas referente às evocações dos enfermeiros e médicos, foi construída a Figura 2, que ilustra o conjunto das palavras evocadas primeiramente e mais frequentemente no núcleo central, separadas por categoria profissional, seguidas pela zona de contraste, primeira e segunda periferias.

De acordo com as evocações referidas pela categoria profissional médica, o núcleo central foi composto por: cuidado, trazendo a dimensão imagética da representação, como se ocorresse um espelhamento do próprio objeto, e adesão-tratamento, que traz a sua dimensão prática. Pode-se inferir que estas palavras evocadas dizem respeito ao trabalho profissional no desempenho de suas atividades em que o acolhimento faz parte da política de humanização do Sistema Único de Saúde. Para estes profissionais, evidencia-se que é necessária a adesão ao tratamento medicamentoso para que haja êxito no cuidado em saúde.

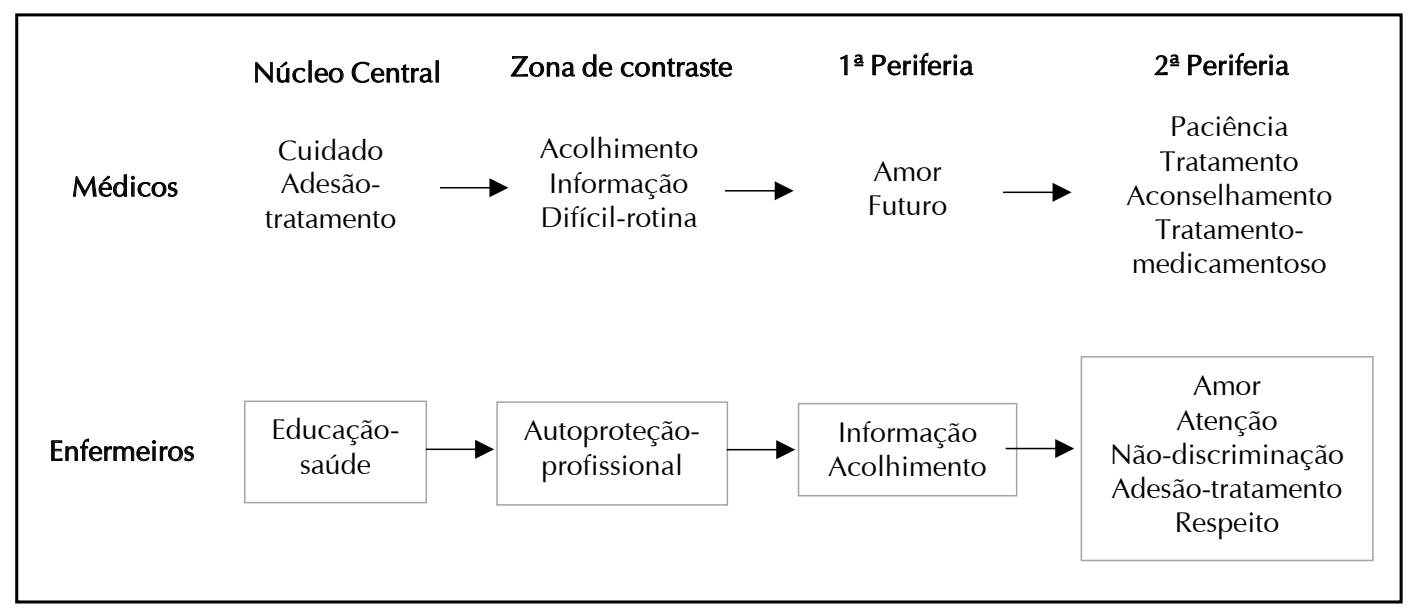

FIGURA 2: Esquema dos termos evocados pelos entrevistados frente ao termo indutor cuidar da pessoa com HIV/ AIDS. Rio de Janeiro, 2014. 
Já no núcleo central da categoria dos enfermeiros, a palavra mais prontamente evocada e com maior frequência foi educação-saúde, conforme a Figura 2. Fica evidente que, para os enfermeiros que compõem este estudo, não é possível cuidar sem educar: por meio da troca de conhecimentos, pode-se auxiliar a PVHA no enfrentamento da síndrome e das barreiras que ela pode provocar. $\mathrm{Na}$ educação em saúde, o profissional transmite conhecimentos ao cliente para que aprenda mais sobre sua condição de saúde, tenha curiosidade de conhecer mais sobre a infecção pelo HIV, como protegerse e cuidar de si, adaptando-se ao tratamento com o objetivo de alcançar mais qualidade de vida. Porém, não emergiram termos como consulta de enfermagem, uma ação privativa do enfermeiro e que se constitui numa ação essencial de cuidado prestado por este profissional.

A zona de elementos de contraste, no quadrante inferior esquerdo, traz as palavras citadas por poucos sujeitos, porém são avaliadas por eles como importantes $^{12,13}$. Na zona de contraste das palavras evocadas pelos médicos, foram apontados os termos acolhimento, difícil-rotina e informação. Já para os enfermeiros, na zona de contraste, há o termo autoproteção-profissional.

A palavra acolhimento indica uma dimensão afetiva, está envolvida com a receptividade e com a escuta ao sujeito que recebe as ações de cuidado ${ }^{14,15}$. Os termos difícil-rotina e informação estão relacionados ao dia a dia do profissional que lida com as dificuldades diárias como infraestrutura precária para o atendimento de forma correta, dificuldades para solicitar exames e revelar o diagnóstico ao cliente, assim como conduzir o tratamento medicamentoso, estimular a aceitação desse diagnóstico e desse tratamento por parte do cliente, que necessita de informações para conhecer mais sobre a AIDS.

O termo difícil-rotina pode demonstrar uma dimensão imagética negativa da representação do cuidado. O termo informação pode estar relacionado ao termo educação-saúde, evocado no núcleo central pelos enfermeiros, mas de outra forma. A palavra informar trata de organizar dados, informações e transmiti-las a outra pessoa, o que difere de educar quando existe diálogo com o outro, havendo troca de saberes. O termo informar pode estar relacionado, para os médicos, à dimensão prática de seu trabalho ao informar sobre o diagnóstico, a prescrição medicamentosa, os exames laboratoriais e o sistema de saúde.

O termo autoproteção-profissional relaciona-se também ao cotidiano de trabalho dos enfermeiros que, em sua prática, realizam procedimentos invasivos e mantêm um contato muito próximo ao cliente vivendo com HIV/AIDS, o que pode gerar maior representação da possibilidade de contaminação durante o trabalho se não utilizar os equipamentos de proteção pessoal no trabalho, pelo risco de contaminação em caso de acidentes em que haja contato com sangue e secreção contaminados. Atualmente, pelo grande número de clientes convivendo com o HIV/AIDS de forma crônica, o termo autoproteção-profissional pode estar ancorado na memória social da AIDS, que inicialmente era vinculada ao medo e à morte ${ }^{16}$, entre outras representações. Ou, ainda, à ênfase na biossegurança trazida a partir da epidemia de AIDS $^{17}$.

A zona periférica comporta palavras que foram evocadas mais tardiamente e com maiores ou menores frequências, expressando os elementos representacionais que podem ser associados ao contexto de vida e às práticas sociais, sendo, portanto, instáveis, permitindo a variabilidade interindividual da representação $0^{14}$.

A primeira periferia, para os médicos, foi composta pelos termos amor e futuro. O termo amor relaciona-se à dimensão afetiva da representação. $\mathrm{O}$ termo futuro pode indicar uma dimensão imagética da representação, vislumbrando que, hoje, as PVHA podem pensar no futuro por meio da adesão ao tratamento e que é necessário ter paciência para suportar todos os efeitos da terapia medicamentosa e os que a própria infecção acarreta ao organismo.

$\mathrm{Na}$ primeira periferia, para os enfermeiros, emergiram os termos acolhimento e informação. Mais prontamente, apareceu o termo acolhimento, que está relacionado à prática de atendimento à PVHA na qual o acolhimento é uma ferramenta essencial no cuidado a este cliente e a todos aqueles que submetem-se ao teste HIV. O acolhimento é uma ação técnico-assistencial a fim de atender a todos os que procuram os serviços de saúde, sendo, portanto, acolhidos e ouvidos quanto às suas necessidades, o que implica um atendimento prestado com resolutividade ${ }^{15}$. O núcleo central, educaçãosaúde, é reforçado pela atitude relacionada aos termos informação e não discriminação, localizados na primeira e segunda periferias, respectivamente.

$\mathrm{Na}$ segunda periferia, que corresponde ao quadrante inferior direito, estão as cognições menos frequentes e importantes para a representação, as quais estão sujeitas a uma mudança mais frequente com a função de proteger o núcleo central e manter a representação.

A segunda periferia da evocação médica é composta pelos termos aconselhamento, paciência, tratamento e tratamento-medicamentoso, que reforçam a importância das ações terapêuticas realizadas para a adesão do cliente ao tratamento. $\mathrm{O}$ aconselhamento enfatiza a necessidade desta adesão ao tratamento medicamentoso, de acordo com o avanço da infecção pelo HIV no organismo. Estes termos, também, recebem uma forte influência da dimensão prática dos profissionais, por meio do tratamento farmacológico e das diretrizes da Política Nacional no combate às DST/AIDS. Para os enfermeiros, a segunda periferia é composta por adesão-tratamento, amor, atenção, não discriminação e respeito, reforçando a importância da adesão ao tratamento e o respeito à pessoa vivendo com HIV/AIDS, relacionado ao ato de cuidar. 
Quanto à caracterização do provável núcleo central para ambas as categorias profissionais, pode-se dizer que este possui uma dimensão funcional em sua ativação. Esta característica relaciona-se à proximidade dos sujeitos com o objeto social investigado, em que são focalizados os elementos mais importantes para a realização de determinada tarefa, neste caso, o cuidado em saúde à pessoa vivendo com HIV/AIDS ${ }^{12,13}$.

De acordo com os prováveis núcleos centrais para médicos e enfermeiros, pode-se extrair que os médicos representam que o cuidado deve ser realizado por meio do acolhimento ao cliente e de suas necessidades, sendo imperativa a adesão ao tratamento para que este cuidado em saúde seja efetivado. Para os enfermeiros, o núcleo central de sua representação sobre o cuidado demonstra que o cuidado está diretamente ligado à educação em saúde, sendo o ponto de partida para qualquer ação em saúde o aconselhamento, os esclarecimentos e informações sobre o HIV/AIDS e tudo que engloba esta temática, como a prevenção e o tratamento, por meio da comunicação e do relacionamento interpessoal. Estas representações podem estar atreladas à formação dos profissionais, o médico com a formação em que o cuidado tem caráter curativo e o enfermeiro, que é formado para cuidar por meio da educação, escuta e prevenção.

Portanto, é possível dizer, pelas interpretações das evocações dos profissionais que participaram deste estudo, que o núcleo central da representação do cuidado em saúde à PVHA para os médicos pauta-se na prescrição medicamentosa, baseado nos eixos do Ministério da Saúde, vivenciados na rotina laboral e com o foco no tratamento medicamentoso, ou seja, uma das bases da medicina no intuito de manter ou alcançar a saúde. Já para os enfermeiros, o núcleo central da representação do cuidado à pessoa com HIV/ AIDS está pautado na educação em saúde, ou seja, no relacionamento e comunicação com o outro, um papel próprio do enfermeiro, por intermédio da preocupação com a autoproteção profissional, atitudes e ações de cuidado. O núcleo central das representações sociais do cuidado para ambas as categorias profissionais baseia-se no saber reificado pela formação profissional.

\section{Conclusão}

Por meio deste estudo, identificaram-se elementos positivos como prováveis componentes do núcleo central das representações sociais de enfermeiros e médicos acerca do cuidado à pessoa que vive com HIV/AIDS. Os cognemas acolhimento, adesão-tratamento, futuro e informação estão relacionados às ações e recomendações do Ministério da Saúde e às características essenciais das profissões de medicina e enfermagem.

Observou-se que enfermeiros e médicos apresentam conteúdos divergentes nos núcleos centrais acerca das representações do cuidado em saúde à pessoa que vive com HIV/AIDS. As evocações dos médicos revelam o cuidado em saúde baseado na adesão ao tratamento como eixo principal de sua ação. Por outro lado, os enfermeiros expressam como núcleo central do cuidado em saúde o termo educação em saúde, sendo as ações educativas bases para o cuidado de enfermagem no grupo estudado. Ações privativas do enfermeiro, como a consulta de enfermagem, não foram citadas.

As representações que emergiram neste estudo podem estar ancoradas nas bases da formação dos profissionais com o saber científico. Porém, não é possível afirmar que as representações de médicos e enfermeiros sobre o cuidado em saúde à PVHA são distintas, pois são necessárias outras técnicas confirmatórias da centralidade para constatar a existência de diferentes representações.

Ressalta-se, ainda, a importância de realizar estudos que reduzam a pressão normativa para uma melhor compreensão do objeto estudado.

\section{REFERÊNCIAS}

1.Villarinho MV, Padilha MI, Berardinelli LMM, Borenstein MS, Meirelles BHS, Andrade SR. Políticas públicas de saúde face à epidemia da AIDS e a assistência às pessoas com a doença. Rev Bras Enferm. [Internet]. 2013 [citado em: 20 nov 2014]; 66: 271-7. Disponível em: http://www.scielo.br/scielo.php?script $=$ sci_ arttext\&pid=S0034-71672013000200018\&lng=en.

2.Jodelet D. Representações sociais: um domínio em expansão. In: Jodelet $\mathrm{D}$, organizadora. As representações sociais. Rio de Janeiro: EdUerj; 2001: 17-44.

3.Formozo GA, Oliveira DC. Representações sociais do cuidado prestado aos pacientes soropositivos ao HIV. Rev Bras Enferm, 2010; 63: 230-7.

4.Luz PM, Miranda KCL. As bases filosóficas e históricas do cuidado e a convocação de parceiros sexuais em HIV/ AIDS como forma de cuidar. Ciênc saúde coletiva. 2010; 15 (Supl. 1): 1143-8.

5.Ministério da Saúde (Br). Lei no 9.313, de 13 de novembro de 1996. Dispões sobre a distribuição gratuita de medicamentos aos portadores do HIV e doentes de AIDS [legislação na internet]. Diário Oficial da União. Brasília, 1996 [citado em 10 jul 2014]. Disponível em: http://www6.senado.gov.br/legislacao/ListaTextoIntegral. action?id=122864.

6.United Nations Program on HIV/AIDS. The gap report. Joint United Nations Programme on HIV/AIDS (UNAIDS). Genebra (Swi): UNAIDS; 2014. [cited in 2014 Nov 20] Disposable in: http://www.unAIDS.org/en/ media/unAIDS/contentassets/documents/unAIDSpublication/2014/UNAIDS_Gap_report_en.pdf.

7.Sadala MLA, Marques SA. Vinte anos de assistência a pessoas vivendo com HIV/AIDS no Brasil: a perspectiva de profissionais da saúde. Cad Saúde Pública [online], 2006; 22: 2369-78. [citado em 20 nov 2014] Disponível: http:// www.scielo.br/scielo.php?script=sci_arttext\&pid=S0102$311 X 2006001100011 \& \operatorname{lng}=$ en\& $^{-}{ }^{-} \mathrm{m}=$ iso. 
8.Formozo GA, Oliveira DC, Costa TL, Gomes AMT. As relações interpessoais no cuidado em saúde: uma aproximação ao problema. Rev enferm UERJ. 2012; 20: 124-7. [citado em 20 nov 2014] Disponível em: http:// www.facenf.uerj.br/v20n1/v20n1a21.pdf .

9.Sá CP. A Construção do objeto de pesquisa em representações sociais. Rio de Janeiro: Eduerj; 1998.

10.Sá CP. As representações sociais na história recente e na atualidade da psicologia social. In: Jacó-Vilela AM, Ferreira AAL e Portugal FT, organizadores. História da psicologia: rumos e percursos. Rio de Janeiro: Nau Ed.; 2007. p. 587-606. 11.Oliveira DC, Marques SC, Gomes AMT, Teixeira MCTV. Análise das evocações livres: uma técnica de análise estrutural das representações sociais. In: Paredes AS. Perspectivas teórico-metodológicas em representações sociais. João Pessoa (PB): Editora Universitária UFPB; 2005 p. 573-603.

12.Abric J-C. A abordagem estrutural das representações sociais: desenvolvimentos recentes. In: Campos PHF, Loureiro MCS. Representações sociais e práticas educativas. Goiânia (GO): Ed. UCG; 2003 p. 37-57.

13.Costa TL, Oliveira DC, Formozo GA, Gomes AMT. Análise estrutural das representações sociais da terapia antirretroviral entre pessoas que vivem com HIV/AIDS: possibilidades de convivência, normatividade e ressignificação. Psicologia e Saber Social. 2013; 2: 104-14.

14.Oliveira DC, Gomes AMT, Marques SC. Análise estatística de dados textuais na pesquisa das representações sociais: alguns princípios e uma aplicação ao campo da saúde. In: Menin MSS, Shimizu AM, organizadores. Experiência e representação social: questões teóricas e metodológicas. São Paulo: Casa do Psicólogo; 2005 p. 157-200.

15.Falk MLR, Falk JW, Oliveira FA, Motta MS. Acolhimento como dispositivo de humanização: percepção do usuário e do trabalhador em saúde. Rev APS. 2010; 13: 4-9. [citado em 20 nov 2014] Disponível em: http://aps. ufjf.emnuvens.com.br/aps/article/view/350/291.

16. Oliveira DC. Construção e transformação das representações sociais da aids e implicações para os cuidados de saúde. Rev Latino-Am Enfermagem [Internet]. 2013 [citado em 20 out 2014];21 (esp):[10 telas]. Disponível em: http://www.scielo.br/pdf/rlae/v21nspe/pt_34.pdf. 17.Gir E, Prado MA, Canini SRMS, Hayashida M. O impacto da AIDS na prática de enfermagem: um problema de saúde pública. DST Jornal brasileiro de doenças sexualmente transmissíveis. 2005; 17: 39-43. [citado em 20 nov 2014] Disponível em: http://www.dst.uff.br/ revista17-1-2005/oimpactodaaids.pdf. 